\title{
MICROCEFALIA EN RECIÉN NACIDOS EN ESTABLECIMIENTOS DE SALUD DE NIVEL II Y III DEL MINISTERIO DE SALUD DE PERÚ
}

\author{
Gabriela Santos-Antonio ${ }^{1, a}$, Fredy Canchihuamán ${ }^{1,2, b}$, Lucio Huamán-Espino ${ }^{1, c}$, Juan Pablo Aparco ${ }^{1, d}$, Jenny Pillaca ${ }^{1, e}$, \\ Daniel Guillén-Pinto ${ }^{2,3, \mathrm{f}}$ Ernesto Gozzer ${ }^{2, \mathrm{~g}}$
}

\begin{abstract}
RESUMEN
Objetivos. Determinar la proporción de microcefalia en recién nacidos en establecimientos de salud (EESS) de nivel II y III del Ministerio de Salud (MINSA) de Perú durante el periodo 2005-2013. Materiales y métodos. Se realizó un análisis secundario de las bases de datos del Sistema Informático Perinatal durante 2005-2013. La identificación de microcefalia se realizó aplicando los criterios de la Organización Mundial de la Salud (OMS), de Fenton, mixto (OMS-Fenton) y de proporcionalidad. Se estimaron las proporciones y tasas de microcefalia por 10000 nacidos vivos (NV) por regiones y se comparó la concordancia, considerando al parámetro de OMS como referencia. Resultados. La proporción de microcefalia durante el 2005 a 2013 fue de 3,4\%, la tasa promedio de microcefalia fue de 335 por 10000 NV según el criterio de OMS. El parámetro mixto mostró una concordancia sustancial (Kappa de 0,635), mientras que el de proporcionalidad mostró una concordancia razonable (Kappa de 0,298). Conclusiones. La proporción de microcefalia en EESS de nivel II y III del MINSA fue mayor a lo reportado en otros países de la región antes de la aparición del Zika en las Américas. Las variaciones de las frecuencias observadas con las de otros países y entre las regiones peruanas, se podrían explicar por diferentes factores, como la técnica de medición del perímetro cefálico, captura de datos, factores constitucionales y determinantes sociales. Sugerimos estandarizar las mediciones y su registro, uniformizar los criterios de diagnóstico y establecer las estrategias sanitarias para fortalecer la vigilancia epidemiológica de las causas de la microcefalia.
\end{abstract}

Palabras clave: Microcefalia; Perímetro cefálico; Prevalencia; Métodos; Diagnóstico; Zika; Perú (fuente: DeCS BIREME).

\section{MICROCEPHALY IN NEWBORNS IN LEVEL II AND III HEALTH FACILITIES OF THE MINISTRY OF HEALTH OF PERU}

\begin{abstract}
Objectives. To determine the ratio of microcephaly in newborns in level II and III health facilities of the Ministry of Health (MINSA) of Peru for the period 2005-2013. Materials and Methods. A secondary analysis of the databases of the Perinatal Information System was carried out during 2005-2013. Microcephaly was identified applying World Health Organization (WHO), Fenton, mixed (WHO-Fenton), and proportionality criteria. The ratios and indices of microcephaly were estimated per 10,000 live births (LB) by region and concordance was compared, using the WHO parameter as a reference. Results. The ratio of microcephaly during 2005 to 2013 was $3.4 \%$, the average rate of microcephaly was 335 per 10,000 LBs according to the WHO criterion. The mixed parameter showed a substantial concordance (Kappa of 0.635), while the proportionality parameter showed a reasonable concordance (Kappa of 0.298). Conclusions. The ratio of microcephaly in MINSA Level II and III health facilities was higher than that reported in other countries in the region before the appearance of Zika in the Americas. The frequency variations observed with those of other countries and among Peruvian regions could be explained by different factors, such as the technique for measuring head circumference, data capture, constitutional factors, and social determinants. We suggest standardizing measurements and their recording, harmonizing diagnostic criteria, and establishing health strategies to strengthen the epidemiological surveillance of the causes of microcephaly.
\end{abstract}

Keywords: Microcephaly; Cephalic perimeter; Prevalence; Methods; Diagnosis; Zika; Peru (source: MeSH NLM).

\footnotetext{
Instituto Nacional de Salud. Lima, Perú.

Universidad Peruana Cayetano Heredia. Lima, Perú.

Hospital Cayetano Heredia. Lima, Perú.

Obstetra; ${ }^{\mathrm{b}}$ médico cirujano, $\mathrm{PhD} ;{ }^{\mathrm{c}}$ sociólogo, $\mathrm{PhD} ;{ }^{\mathrm{d}}$ nutricionista, $\mathrm{MSc} ;{ }^{\mathrm{e}}$ estadístico; ${ }^{\mathrm{f}}$ neurólogo pediatra, $\mathrm{PhD},{ }^{\mathrm{g}}$ médico cirujano, $\mathrm{MSc}$.
}

Recibido: 13/11/2018 Aprobado:22/05/2019 En línea: 28/06/2019

Citar como: Santos-Antonio G, Canchihuamán F, Huamán-Espino L, Aparco JP, Pillaca J, Guillén-Pinto D, et al. Microcefalia en recién nacidos en establecimientos de salud de nivel II y III del Ministerio de Salud de Perú. Rev Peru Med Exp Salud Pública. 2019;36(2):222-30. doi: http://dx.doi.org/10.17843/rpmesp.2019.362.4045. 


\section{INTRODUCCIÓN}

La medición del perímetro cefálico al nacimiento y posnatal es una medida fácil de realizar, no invasiva y de bajo costo. Forma parte de la evaluación integral del niño y permite identificar oportunamente casos de microcefalia y macrocefalia, relacionados con posibles daños al sistema nervioso neonatal ${ }^{(1)}$ y determinar variaciones durante la infancia temprana como signos de alerta de trastornos del desarrollo (2).

Las complicaciones severas asociadas a la infección por el virus del Zika como la microcefalia y el síndrome de GuillainBarré motivaron que la Organización Mundial de la Salud (OMS) declare esta enfermedad como una emergencia de salud pública de importancia internacional ${ }^{(3)}$. En este contexto, la medición del perímetro cefálico al nacimiento recobró relevancia y la detección de casos de microcefalia fue considerada como una actividad crítica de los sistemas de vigilancia en los países con o en riesgo de Zika ${ }^{(4)}$. Una particular experiencia fue desarrollada al noreste de Brasil, a finales del 2015 , donde se detectó una variación de la tasa de microcefalia de 40 a 80 casos por 10000 nacidos vivos (NV) ${ }^{(5)}$

Pese a que la vigilancia de la microcefalia es un factor importante para detectar nuevos riesgos de salud, las determinaciones de su magnitud han sido variables por diversos factores como el uso de distintos parámetros y puntos de corte ${ }^{(6)}$, el uso de diferentes técnicas de medición del perímetro cefálico y la omisión de la medición al nacer o la mala calidad del registro ${ }^{(7)}$. Estos y otros factores pueden afectar la efectividad de los sistemas de vigilancia y es por ello que se ha enfatizado en la necesidad de uniformizar los criterios de medición y registro ${ }^{(8)}$.

A pesar de la necesidad de contar con esta información, nuestro país no tiene datos sobre la proporción de microcefalia a nivel nacional o regional; aunque dos hospitales de Lima reportaron previamente sus tasas $(9,10)$. En ese contexto, el presente estudio tuvo como objetivo determinar la proporción de microcefalia en establecimientos de salud (EESS) de nivel II y III del Ministerio de Salud (MINSA) de Perú durante 2005-2013, a partir de datos del Sistema Informático Perinatal (SIP 2000) y estimar el grado de concordancia entre cuatro criterios diagnósticos de microcefalia.

\section{MATERIALES Y MÉTODOS}

\section{DISEÑO DEL ESTUDIO}

Se realizó un análisis secundario de datos del Sistema Informático Perinatal (SIP 2000) del 2005 al 2013. Estos datos fueron recolectados para el estudio titulado «Asociación entre el control prenatal y peso-longitud de

\section{MENSAJES CLAVE}

Motivación para realizar el estudio. En Perú, no existe información sobre la magnitud de la microcefalia en recién nacidos.

Principales hallazgos. La proporción de microcefalia en establecimientos de salud de nivel II y III del Ministerio de Salud de Perú durante el período 2005-2013 fue de 3,4\%, esta proporción es mayor respecto a la de otros países de la región.

Implicancias. Los resultados sirven como un parámetro de base para comparar y determinar variaciones en el tiempo. Es importante una determinación precisa de casos, frecuencias y tendencias para una detección oportuna de riesgos y una respuesta rápida; no obstante, existen retos para mejorar la precisión.

los recién nacidos en establecimientos de salud nivel II y III del MINSA» (11). Los EESS de nivel II y III son hospitales que atienden partos (con diferentes niveles de resolución) a diferencia del nivel I, que son puestos o centros de salud ${ }^{(12)}$.

\section{FUENTE DE INFORMACIÓN}

La base de datos del estudio primario fue generada usando los registros del SIP 2000 para el periodo 2005 al 2013 de la mayoría de los EESS nivel de atención II y III del MINSA en el Perú. De los 154 EESS de nivel II y III existentes, 28 no registraban información alguna, tres utilizaban un software diferente, uno rechazó entregar su información. Finalmente 122 EESS entregaron sus bases de datos $(79,2 \%$ del total). El SIP 2000 registra los datos de las atenciones realizadas a la gestante durante el embarazo, parto y puerperio inmediato tomadas de la historia clínica materno perinatal. La información se encuentra en cada EESS; debido a que no existe un sistema de acopio a nivel central. Al momento de recogerse los datos para el estudio primario, se firmaron cartas de compromiso ético a fin de preservar la confidencialidad de los datos. Esta información está bajo la custodia del Instituto Nacional de Salud (INS) de Perú.

La base de datos inicial para el análisis secundario tenía 1327725 registros de gestantes cuyos partos correspondían del 2005 al 2013. Se excluyeron los registros con inconsistencia entre la edad gestacional por fecha de última regla (FUR) y la edad por examen físico (diferencia mayor $a \pm 2$ semanas), los neonatos prematuros 0 pretérminos ( $<37$ semanas) y postérmino ( $\geq 42$ semanas), los óbitos y natimuertos, los que no tenían dato sobre sexo y los que no tenían datos sobre perímetro cefálico. Asimismo, se retiraron los registros de perímetro cefálico mayores de \pm 5 desviaciones estándar (DE). La base final para el análisis estuvo conformada por 548919 registros validos (Figura 1).

\section{DEFINICIÓN DE VARIABLES}

La microcefalia se clasificó utilizando cuatro criterios: 1) parámetro de la OMS que tiene un único punto de corte 


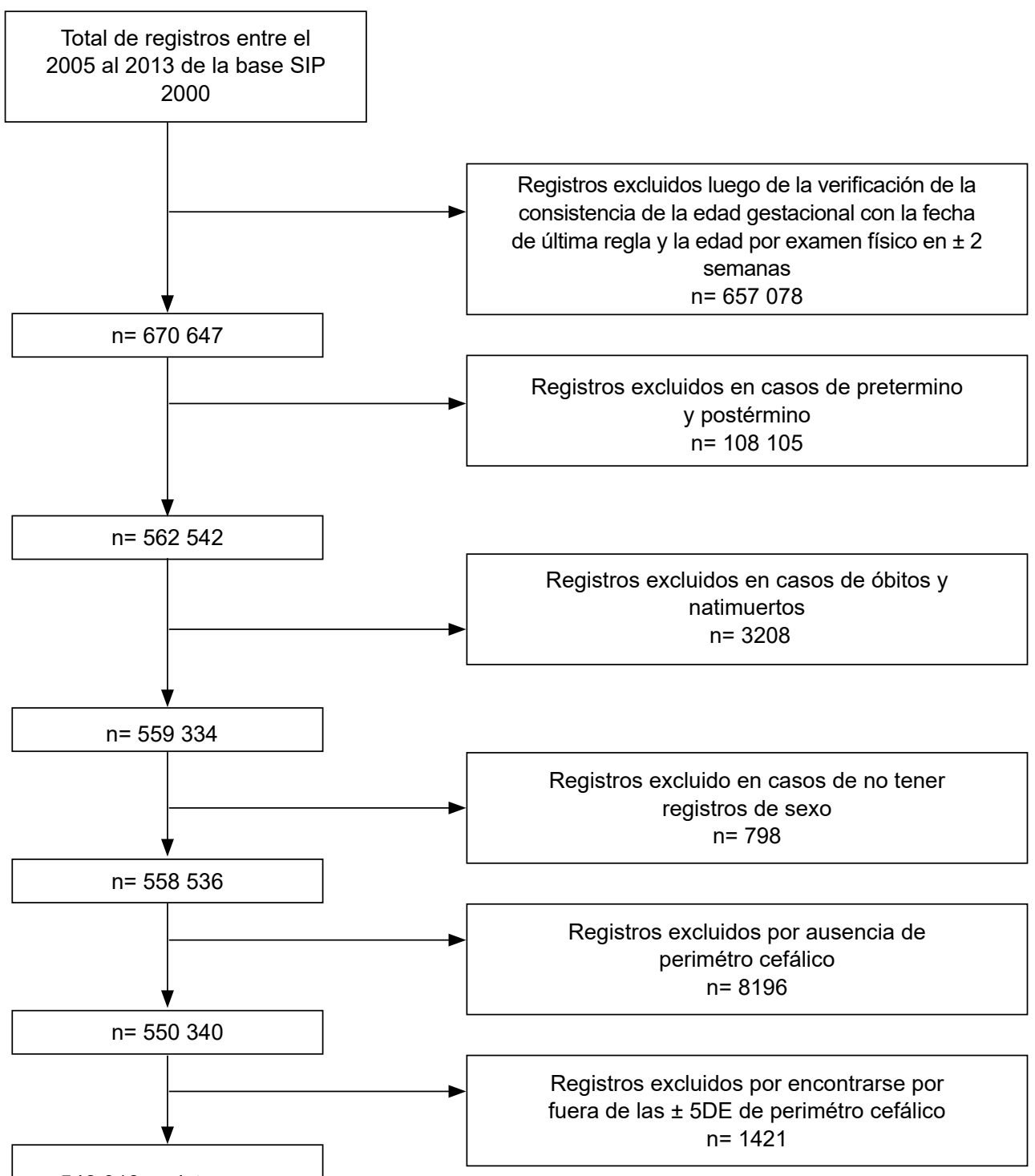

548919 registros que

ingresan al análisis de datos

Figura 1. Flujograma de selección de los registros para la estimación de la proporción de microcefalia

para todos los niños a término y determina microcefalia cuando el perímetro cefálico, según sexo, se ubica por debajo de -2 DE de la curva de referencia (usado para vigilancia en salud pública) y $-3 \mathrm{DE}$ para los casos de microcefalia severa ${ }^{(13)}$. 2) Curvas de Fenton que tiene valores desde las 22 semanas hasta las 50 semanas gestacionales y aplica como punto de corte $-2 \mathrm{DE}$ según sexo y edad gestacional del recién nacido ${ }^{(14)}$. 3) Criterio de proporcionalidad, en el que cada neonato es clasificado como microcefálico si el perímetro cefálico se encuentra por debajo del valor estimado según la siguiente formula: [(Longitud/2)+10)-2] (15). 4) Criterio mixto que combina los criterios de Fenton y OMS, en el presente estudio se propuso y aplicó el criterio mixto considerando que en el contexto de la alerta del Zika la OMS menciona que los valores (puntos de corte de microcefalia) deben estandarizarse según edad gestacional para una mejor vigilancia (13); además, el American Collegue of Obstericians and Gynaecologist (ACOG) propone una subdivisión de los niños a término, basado en la mayor presencia de morbilidad y mortalidad, recomendando tener mayor vigilancia en los niños de 37 , 38 y 39 semanas ${ }^{(16)}$. Por ello, los autores elaboramos un criterio mixto que consiste en aplicar las curvas de Fenton en los recién nacidos de edades gestacionales de 37, 38 y 39 semanas y los parámetros de OMS para los recién nacidos de 40 y 41 semanas.

Además, se analizaron como variables relacionadas a la madre: edad en años $(<20,20-35$ y $>35)$, nivel de educación (analfabeta/primaria, secundaria, superior), índice de 
masa corporal (IMC) preconcepcional (delgada, normal, sobrepeso y obesa), paridad (primigesta, segundigesta y multípara/granmultípara), número de controles prenatales ( $<6$ y $\geq 6$ controles), región (costa, sierra y selva) y como variables relacionadas a los neonatos: edad gestacional al nacimiento, sexo, tipo de parto (vaginal y cesárea) y clasificación del recién nacido según el peso para la madurez estimada (pequeño, adecuado y grande para edad gestacional).

\section{CONSTRUCCIÓN DE INDICADORES}

Los indicadores se elaboraron en base al número de recién nacidos vivos a término. Para la estimación de la tasa de microcefalia por año se consideró en el numerador los casos de microcefalia y el denominador el total de recién NV con datos válidos, todo esto multiplicado por 10 000. Para la estimación de la proporción se usó como numerador los casos de microcefalia detectados y como denominador el total de recién nacidos vivos con datos válidos por 100 .

\section{ANÁLISIS DE DATOS}

Para el análisis estadístico se construyeron tablas de distribución de frecuencias para las variables de la madre y del neonato, además se estimaron las proporciones, tasas de microcefalia por regiones y la proporción de microcefalia promedio por año. El análisis de concordancia entre los criterios de clasificación de microcefalia se realizó aplicando la prueba Kappa de Cohen, usando los siguientes cortes: 0,00 «pobre», de 0,01 a 0,20 «leve», de 0,21 a 0,40 «razonable», entre 0,41 y 0,60 «moderada», de 0,61 a 0,80 «sustancial» y de 0,81 a 1 "casi perfecta» concordancia (17). Los datos se analizaron en el programa estadístico STATA versión 14.

\section{CONSIDERACIONES ÉTICAS}

Para la realización del estudio no se requirió de la aprobación de un Comité de Ética por tratarse de un análisis de datos secundarios cuyo estudio principal contó con la aprobación del Comité de Ética en Investigación del INS. En el referido estudio principal se firmaron cartas de compromiso para la salvaguarda de la información y privacidad de las personas. En el análisis se utilizaron códigos y no datos personales.

\section{RESULTADOS}

En el estudio se incluyeron 548919 recién nacidos del Sistema Informático Perinatal (SIP 2000) de 122 EESS de nivel II y III de diferentes regiones del Perú, en el periodo 2005-2013.

\section{CARACTERÍSTICAS DE LA POBLACIÓN}

El $50 \%$ las madres procedían de la costa, en su mayoría tenían nivel de educación secundaria y un rango de edad predominante entre 20 a 35 años (Tabla 1). Además, cerca del $70 \%$ de madres tenía un IMC pregestacional normal, un $40 \%$ era primigesta y el $67 \%$ culminó el embarazo con seis o más controles prenatales. En la misma tabla se aprecia que el $36 \%$ de neonatos nacieron con 38 semanas

Tabla 1. Características de las madres y de los neonatos registrados en el Sistema de Información Perinatal, 2005-2013

\begin{tabular}{|c|c|c|}
\hline Características de las madres & $\mathbf{n}$ & $\%$ \\
\hline \multicolumn{3}{|l|}{ Región } \\
\hline Costa & 281356 & 51,7 \\
\hline Sierra & 158373 & 29,2 \\
\hline Selva & 103495 & 19,1 \\
\hline \multicolumn{3}{|l|}{ Edad (años) } \\
\hline$<20$ & 117823 & 21,8 \\
\hline $20-35$ & 374944 & 69,4 \\
\hline$>35$ & 47887 & 8,9 \\
\hline \multicolumn{3}{|l|}{ Nivel de educación } \\
\hline Analfabeta/Primaria & 105732 & 19,8 \\
\hline Secundaria & 327967 & 61,4 \\
\hline Superior & 100679 & 18,8 \\
\hline \multicolumn{3}{|l|}{ IMC preconcepcional } \\
\hline Delgadez & 14912 & 3,2 \\
\hline Normal & 320985 & 69,3 \\
\hline Sobrepeso & 88326 & 19,1 \\
\hline Obesidad & 39076 & 8,4 \\
\hline \multicolumn{3}{|l|}{ Clasificación de paridad } \\
\hline Primigesta & 215376 & 40,5 \\
\hline Segundigesta & 141266 & 26,5 \\
\hline Multípara/Gran multípara & 175530 & 33,0 \\
\hline \multicolumn{3}{|l|}{ Número de CPN } \\
\hline$<6$ & 143878 & 33,0 \\
\hline$\geq 6$ & 291752 & 67,0 \\
\hline \multicolumn{3}{|l|}{ De los neonatos } \\
\hline \multicolumn{3}{|l|}{ Edad gestacional (semanas) } \\
\hline 37 & 134638 & 24,8 \\
\hline 38 & 196408 & 36,2 \\
\hline 39 & 139965 & 25,8 \\
\hline 40 & 56701 & 10,4 \\
\hline 41 & 15512 & 2,9 \\
\hline \multicolumn{3}{|l|}{ Sexo } \\
\hline Masculino & 272459 & 50,2 \\
\hline Femenino & 270765 & 49,8 \\
\hline \multicolumn{3}{|l|}{ Tipo de parto } \\
\hline Normal & 385620 & 71,1 \\
\hline Cesárea & 155716 & 28,7 \\
\hline Instrumentado & 1245 & 0,2 \\
\hline \multicolumn{3}{|l|}{ Clasificación del recién nacido* } \\
\hline Pequeño & 34614 & 6,3 \\
\hline Adecuado & 411617 & 82,3 \\
\hline Grande & 62683 & 11,4 \\
\hline
\end{tabular}

CPN: Control prenatal, IMC: Índice de masa corporal

*Según peso para edad gestacional 
de gestación, un $71 \%$ fue parto normal y el $82 \%$ tuvieron adecuado peso para la edad gestacional. El peso promedio de los neonatos fue de $3336,9 \mathrm{~g}$ (DE: $443,1 \mathrm{~g}$ ), la talla fue de $49,8 \mathrm{~cm}$ (DE: $2,1 \mathrm{~cm}$ ) y el perímetro cefálico fue de 34,3 $\mathrm{cm}$ (DE: 1,5 cm).

\section{DISTRIBUCIÓN POR REGIONES, GRUPOS DE INTERÉS Y AÑOS}

En la Tabla 2 se muestra la distribución de los casos, proporción y tasa promedio de microcefalia por $10000 \mathrm{NV}$ según regiones y se aprecia que destacan con mayores tasas Ucayali, Pasco, Huancavelica, Amazonas y Loreto con tasas por encima de 520 casos por $10000 \mathrm{NV}$, mientras que las regiones con menores tasas son Tacna, Arequipa y Moquegua.

También se observa que la proporción de datos perdidos en la estimación fue, en general, de $59 \%$ y que de este total un $50 \%$ fue por datos vacíos y el otro $50 \%$ fue por datos inconsistentes, en tanto que las regiones con mayor pérdida de datos vacíos fueron Cajamarca, Ica y luego Huánuco (pérdidas por encima del $40 \%$ ) y por datos inconsistentes Lambayeque, Ucayali, Loreto y Moquegua (pérdidas por encima del $37 \%)$.

Respecto a subgrupos de interés, en niños pequeños para edad gestacional se encontró que la proporción de microcefalia fue de $11,5 \%$, mientras que en los adecuados para edad gestacional fue de $1 \%$; en madres con embarazo múltiple la proporción fue 3,1\% y en madres con gestación única de 1,6\%; asimismo, en partos vaginales la proporción fue de $1,8 \%$, en tanto que en partos por cesárea fue de $1 \%$ (datos no presentados en las tablas).

Entre el 2005 a 2013 se encontraron 18428 recién nacidos con microcefalia en los 122 establecimientos de salud de nivel II y III incluidos en el estudio. La Figura 2 muestra

Tabla 2. Número de casos, proporción y tasa promedio de microcefalia por 10000 nacidos vivos, según regiones, 2005-2013

\begin{tabular}{|c|c|c|c|c|c|c|c|c|c|c|}
\hline \multirow{3}{*}{ Región } & \multirow{3}{*}{$\begin{array}{c}\text { Total } \\
\mathbf{n} \\
\end{array}$} & \multicolumn{4}{|c|}{ Datos perdidos } & \multirow{2}{*}{\multicolumn{2}{|c|}{ Datos válidos }} & \multirow{2}{*}{\multicolumn{3}{|c|}{ Microcefalia }} \\
\hline & & \multicolumn{2}{|c|}{ Sin datos } & \multicolumn{2}{|c|}{ Inconsistentes $^{\dagger}$} & & & & & \\
\hline & & $\mathrm{n}$ & $\%$ & $\mathrm{n}$ & $\%$ & $\mathrm{n}$ & $\%$ & $n$ & $\%$ & Tasa $^{\ddagger}$ \\
\hline Amazonas* & 9088 & 3449 & 38,0 & 2441 & 26,9 & 3198 & 35,2 & 174 & 5,4 & 520,7 \\
\hline Ancash & 87423 & 24162 & 27,6 & 24229 & 27,7 & 39032 & 44,7 & 935 & 2,4 & 237,4 \\
\hline Apurímac & 17911 & 5602 & 31,3 & 5143 & 28,7 & 7166 & 40,0 & 373 & 5,2 & 509,8 \\
\hline Arequipa & 61005 & 12205 & 20,0 & 17459 & 28,6 & 31341 & 51,4 & 429 & 1,4 & 138,0 \\
\hline Ayacucho & 49550 & 14326 & 28,9 & 14618 & 29,5 & 20606 & 41,6 & 962 & 4,7 & 465,1 \\
\hline Cajamarca* & 22907 & 12401 & 54,1 & 4647 & 20,3 & 5859 & 25,6 & 212 & 3,6 & 468,1 \\
\hline Cusco & 54600 & 15733 & 28,8 & 18236 & 33,4 & 20631 & 37,8 & 686 & 3,3 & 311,7 \\
\hline Huancavelica* & 13427 & 4688 & 34,9 & 4312 & 32,1 & 4427 & 33,0 & 250 & 5,7 & 559,0 \\
\hline Huánuco & 26907 & 10778 & 40,1 & 8244 & 30,6 & 7885 & 29,3 & 345 & 4,4 & 388,8 \\
\hline Ica & 12304 & 5337 & 43,4 & 3919 & 31,9 & 3048 & 24,8 & 53 & 1,7 & 236,7 \\
\hline Junín & 130443 & 39116 & 30,0 & 38521 & 29,5 & 52806 & 40,5 & 2814 & 5,3 & 532,9 \\
\hline La Libertad & 134532 & 46401 & 34,5 & 34652 & 25,8 & 53479 & 39,8 & 1211 & 2,3 & 223,4 \\
\hline Lambayeque* $^{*}$ & 9547 & 3239 & 33,9 & 3882 & 40,7 & 2426 & 25,4 & 94 & 3,9 & 403,3 \\
\hline Lima & 322770 & 103856 & 32,2 & 84470 & 26,2 & 134444 & 41,7 & 3328 & 2,5 & 247,5 \\
\hline Loreto & 57874 & 12410 & 21,4 & 22000 & 38,0 & 23464 & 40,5 & 1265 & 5,4 & 588,2 \\
\hline Madre de Dios* & 17139 & 3637 & 21,2 & 4160 & 24,3 & 9342 & 54,5 & 187 & 2,0 & 197,8 \\
\hline Moquegua & 11836 & 2153 & 18,2 & 4494 & 38,0 & 5189 & 43,8 & 105 & 2,0 & 195,0 \\
\hline Pasco & 20009 & 4299 & 21,5 & 6986 & 34,9 & 8724 & 43,6 & 539 & 6,2 & 613,7 \\
\hline Piura & 75828 & 26451 & 34,9 & 21326 & 28,1 & 28051 & 37,0 & 1174 & 4,2 & 416,6 \\
\hline Puno & 44236 & 9343 & 21,1 & 14717 & 33,3 & 20176 & 45,6 & 454 & 2,3 & 229,5 \\
\hline San Martin & 42357 & 12318 & 29,1 & 11757 & 27,8 & 18282 & 43,2 & 818 & 4,5 & 438,5 \\
\hline Tacna & 37462 & 7077 & 18,9 & 8637 & 23,1 & 21748 & 58,1 & 222 & 1,0 & 103,9 \\
\hline Ucayali & 68570 & 14673 & 21,4 & 26302 & 38,4 & 27595 & 40,2 & 1798 & 6,5 & 651,1 \\
\hline Total & 1327725 & 393654 & 29,7 & 385152 & 29,0 & 548919 & 41,3 & 18428 & 3,4 & 335,7 \\
\hline
\end{tabular}

NV: nacidos vivos con datos válidos

* Regiones que no aportaron con todos los años: Amazonas del 2006 al 2013, Cajamarca del 2006 al 2013, Huancavelica del 2007 al 2013, Lambayeque 2009, 2012 y 2013, Madre de Dios del 2007 al 2013

† Datos inconsistentes: Se consideraron en esta categoría todos los registros con datos errados en fechas, en edad gestacional (inconsistencia de la edad gestacional determinada por fecha de ultima regla en \pm 15 días con edad gestacional al nacimiento por examen físico) y los datos extremos de perímetro cefálico por encima de las $5 \pm$ desviaciones estándar en la variable principal

¥ Promedio por $10000 \mathrm{NV}$ 


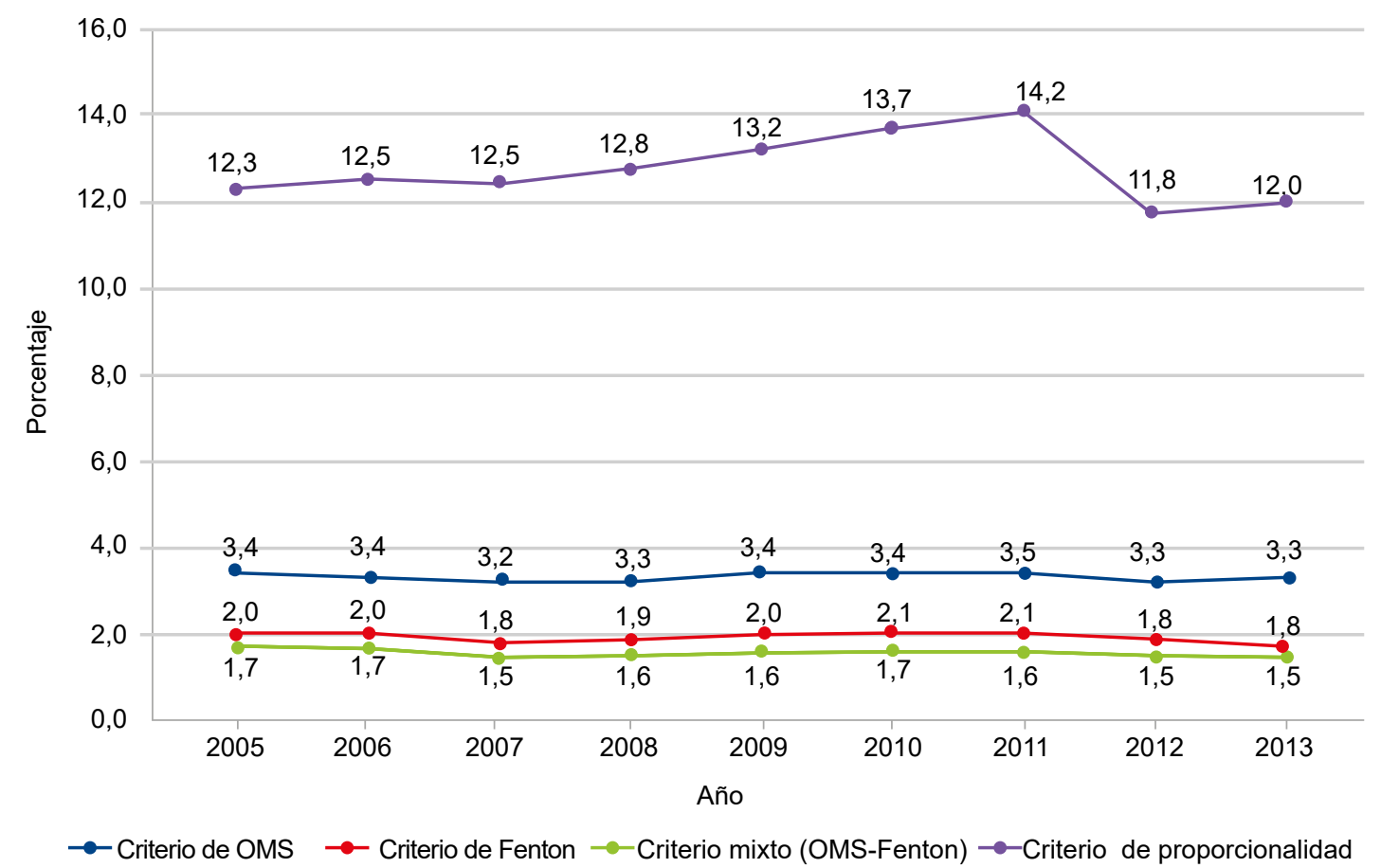

Figura 2. Distribución temporal de neonatos con microcefalia en establecimientos de salud de nivel II y III del Ministerio de Salud, en diferentes regiones del Perú, 2005-2013

la proporción anual de niños con microcefalia y si bien la proporción varió según el criterio de clasificación siendo menor al aplicar el criterio mixto (OMS-Fenton) y mayor con el de proporcionalidad tuvo un patrón similar en este periodo (Figura 2).

\section{CONCORDANCIA ENTRE LOS CUATRO CRITERIOS DE CLASIFICACIÓN DE MICROCEFALIA}

La proporción de microcefalia durante el 2005 al 2013 también varió dependiendo del criterio usado para la clasificación. Al aplicar el parámetro de OMS la proporción promedio para el periodo fue de $3,4 \%$, con Fenton fue de $1,9 \%$, mientras que con el parámetro mixto (OMS-Fenton) resultó en 1,5\% y con el de proporcionalidad fue de 12,8\% (Tabla 3). La concordancia entre el criterio de OMS y Fenton fue moderada (Kappa de 0,590, error estándar 0,003), entre OMS y el criterio mixto (OMS-Fenton) fue sustancial (Kappa de 0,635, error estándar 0,003) y entre OMS y el criterio de proporcionalidad fue razonable (Kappa de 0,298, error estándar 0,002).

\section{DISCUSIÓN}

El estudio constituye, hasta donde conocemos, la primera estimación de proporción de microcefalia en EESS del MINSAde varias regiones del Perú. Los resultados muestran que según el parámetro de OMS la proporción fue de 3,4\% y la tasa de microcefalia fue de 335,6 por 10000 NV. Esta información basal podría utilizarse para estimar variaciones del promedio debido a factores emergentes como el Zika, cuyo ingreso se registró a partir del 2016.

Nuestros resultados difieren considerablemente de las tasas de microcefalia reportadas por la mayoría de los autores. Un estudio realizado en 15 países de Europa encontró una tasa de 1,5 por $10000 \mathrm{NV}(1,1-1,9)$, variando entre países de 0,4 a 4,3 por $10000 \mathrm{NV}$; diferencia explicada por el uso de diferentes puntos de corte (<-3 DS y <-2DS) (18). En Australia, la tasa de microcefalia fue de 5,5 por 10000 NV $(1,82-2,78)$ con <-2 DE para el periodo 1980-2009 (19). En Estados Unidos, la tasa reportada en una revisión de 30 programas de vigilancia fue de 8,7 por $10000 \mathrm{NV}$ con $<-2 D E{ }^{(20)}$, mientras que en la India fue de 2,3 por 10000 NV $(1,8-2,8)^{(21)}$ con <-2 DS y en Japón, en el periodo entre 1979 y 1982 , fue de 0,2 por 10000 NV (22).

Latasa de microcefalia reportadaen países de Latinoamérica antes del inicio de la epidemia de Zika, fue de 2 por 10000 NV $(1,5-2,3)$ en Brasil ${ }^{(23)}$ y de 2,1 por $10000 \mathrm{NV}$ con <-3 DE en Colombia ${ }^{(24)}$. Otro estudio en Brasil, encontró una tasa promedio de 0,5 casos por $10000 \mathrm{NV}$ en el periodo 2000 - 2014 y 5,5 casos por 10000 NV el 2015, siendo la tasa en el noreste de Brasil de 13,9 casos por 10000 NV (25). Un reciente estudio del Grupo ECLAMC (Latin American Collaborative Study of Congenital Malformations) basado en datos de 107 hospitales de 10 países sudamericanos 
Tabla 3. Proporción de microcefalia en recién nacidos según los diferentes criterios considerados

\begin{tabular}{|c|c|}
\hline Criterios & n (\%) \\
\hline \multicolumn{2}{|l|}{ Criterio de OMS } \\
\hline Normal & $530491(96,6)$ \\
\hline \multicolumn{2}{|l|}{ Microcefalia } \\
\hline Total$^{*}(<-2 \mathrm{DE})$ & $18428(3,4)$ \\
\hline Severa $(<-3 D E)$ & $4033(0,7)$ \\
\hline \multicolumn{2}{|l|}{ Criterio de Fenton } \\
\hline Normal & $538240(98,1)$ \\
\hline \multicolumn{2}{|l|}{ Microcefalia } \\
\hline Total* $^{*}(<-2 \mathrm{DE})$ & $10679(1,9)$ \\
\hline Severa $(<-3 D E)$ & $1300(0,2)$ \\
\hline \multicolumn{2}{|c|}{ Criterio mixto (OMS-Fenton) } \\
\hline Normal & $540186(98,5)$ \\
\hline \multicolumn{2}{|l|}{ Microcefalia } \\
\hline Total $^{*}(<-2 \mathrm{DE})$ & $8733(1,5)$ \\
\hline Severa $(<-3 D E)$ & $1042(0,2)$ \\
\hline \multicolumn{2}{|c|}{ Criterio de proporcionalidad ${ }^{\dagger}$} \\
\hline Normal & $474921(87,2)$ \\
\hline Microcefalia ${ }^{\ddagger}$ & $69768(12,8)$ \\
\hline
\end{tabular}

OMS: Organización Mundial de la Salud, DE: Desviación Estándar

* Incluye microcefalia moderada y severa

+ El total de registros analizados es de 544689

‡ Este criterio incluye niños con perímetro cefálico (PC) mayor a $32 \mathrm{~cm}$ (no tienen microcefalia según los parámetros de OMS) pero cuyo tamaño del $\mathrm{PC}$ no es proporcional a la longitud del cuerpo

estimó que la tasa de microcefalia en Sudamérica fue de 4,4 por 10000 para el periodo 2005-2014 (10). Respecto al Perú, la prevalencia de un hospital de referencia del Seguro Social de Salud (EsSalud) ubicado en Lima fue de 3,3 por $10000 \mathrm{NV}$ con <-3 DE. En este mismo estudio se reporta para el noreste de Brasil una tasa de 8,4 por $10000 \mathrm{NV}$.

Dos estudios muestran altas proporciones de microcefalia concordantes con nuestros hallazgos, el primero realizado en un estado del noreste de Brasil que recogió datos de 21 centros de maternidad, encontró una prevalencia de microcefalia de $8,2 \%$ en 16208 neonatos (equivalente a una tasa de 820 por $10000 \mathrm{NV}$ ); además, observaron variaciones en el tiempo con un incremento marcado en casos severos de microcefalia desde el tercer cuatrimestre del $2015^{(5)}$. Otro estudio en Guatemala con datos de un programa de bienestar comunitario en zonas rurales encontró una prevalencia de microcefalia de 6,8\% (26) (aproximadamente una tasa de 680 por 10000 NV). Por otro lado, Quintó et al. ${ }^{(27)}$ aplicando el criterio de OMS, estimaron las prevalencias de microcefalia con datos de un ensayo clínico en Mozambique ${ }^{(28)}$ y datos de un estudio observacional multicéntrico en Colombia, Brasil y Guatemala (29); encontrando proporciones más altas que en nuestro estudio $(3,9 \%, 8,0 \%, 12,3 \%$ y $8,1 \%$, respectivamente). Los autores consideran que estas altas frecuencias se explicarían por la dificultad para determinar la edad gestacional al nacimiento; también mencionan que los pequeños para edad gestacional, que presentaron algún tipo de retardo de crecimiento intrauterino, no se deberían diagnosticar como microcefálicos ${ }^{(27)}$.
Las diferencias de nuestros estimados sobre la magnitud de la microcefalia con los de otros países y las variaciones encontradas en los estimados en algunas regiones del Perú podrían ser producto de diversos factores que afectan la precisión de nuestras mediciones, entre ellos estarían las diferencias en los criterios de diagnóstico y puntos de corte, el tipo de instrumento y las técnicas de medición, el momento de la medición del perímetro cefálico, la calidad de la información registrada, los factores étnicos y geográficos, así como los determinantes sociales, tal como reportan Morris et al (18) y otros autores ${ }^{(30)}$. Asimismo, un estudio en niños de Bélgica y Noruega comparó referencias nacionales con las curvas de crecimiento de OMS y encontró diferencias en el caso del perímetro cefálico ${ }^{(31)}$, por lo que aplicación de las curvas estándares a poblaciones de perímetros cefálicos más pequeños podría sobreestimar la magnitud de la microcefalia.

Respecto a la comparación de concordancias entre los criterios diagnósticos de microcefalia, considerando como estándar el criterio de OMS, encontramos que el parámetro mixto que combina los criterios de OMS y Fenton tuvo una buena concordancia; al respecto propusimos este parámetro mixto debido a que diversos estudios han criticado el uso de una sola medida internacional estándar para valorar las mediciones del perímetro cefálico ${ }^{(30,31)}$ y en algunos casos se han propuesto combinaciones de los parámetros. Las recomendaciones de utilizar puntos de corte fijos para recién nacidos de cualquier edad gestacional ${ }^{(13)}$, han sido cuestionadas porque podrían sobre estimar las prevalencias de microcefalia al no considerar que una proporción importante podría nacer antes de las 40 semanas de gestación. En un sub análisis de nuestra base de datos utilizando ambos criterios para recién nacidos entre 37 y 40 semanas, obtuvimos estimados más altos con la curva de OMS que con la de Fenton. Además, según el criterio de proporcionalidad encontramos que la proporción media de microcefalia para 2005-2013 fue de 12,8\% (tasa de 1280 por $10000 \mathrm{NV}$ aproximadamente) lo que implica que, si bien muchos de estos RN tenían un perímetro cefálico mayor a 32 centímetros, el crecimiento de este perímetro respecto a la longitud de su cuerpo había sido desproporcionado.

Nuestro estudio tiene limitaciones inherentes al uso de datos secundarios. En la determinación de casos de microcefalia utilizamos solo la información del SIP 2000 con medidas del perímetro cefálico, no revisamos otras fuentes primarias o secundarias o las vinculamos con otras fuentes para corroborar, como hicieron otros estudios ${ }^{(4,5)}$. Tampoco pudimos excluir casos de microcefalia por anomalía genética. Lo anterior no fue posible porque el SIP 2000 registra todas las patologías en conjunto y no se interconecta con sistemas de registro perinatal similares como el aplicativo del Centro Latinoamericano de Perinatología (CLAP). 
Otra limitación fue que el $59 \%$ de los registros fueron excluidos por datos vacíos o inconsistentes (ambos en igual proporción) y esto podría afectar los estimados de microcefalia y por tanto la validez y generalización de los resultados a nivel global y por regiones (sesgos de selección); sin embargo, al analizar la distribución temporal a lo largo del periodo 2005-2013 encontramos patrones similares de microcefalia en todos los años. Además, estos datos sólo reflejan la proporción de microcefalia en EESS de nivel II y III del MINSA, no se incluyeron EESS de nivel I, porque estos no registraban datos en el SIP, al momento del estudio y sólo atendían el $19 \%$ de los partos en general.

Las fortalezas del estudio incluyen, la utilización de criterios específicos para eliminar errores y datos inconsistentes, la utilización de criterios diferentes que permiten la comparación con indicadores de otros países, y el uso de la base de datos más grande de registros de información perinatal de EESS de nivel II y III de nuestro país (más de medio millón de recién nacidos en un periodo de nueve años). El uso de similar fuente de datos se ha reportado en otros estudios ${ }^{(25)}$.
En conclusión, el estudio encontró que en EESS de nivel II y III del MINSA la proporción de microcefalia fue de $3,4 \%$, mayor a la reportada por otros países de la región. Posibles diferencias en la técnica de medición, del tipo de instrumento y de los criterios diagnósticos utilizados, las características étnicas y geográficas, así como los efectos de los determinantes sociales de la salud pueden explicar la variabilidad de las altas tasas encontradas. Estos resultados destacan la importancia de estandarizar parámetros de medición y de ampliar los estudios para fortalecer la vigilancia epidemiológica de la microcefalia neonatal, esencial para una detección oportuna de riesgo y una respuesta rápida.

Agradecimientos: Los autores desean agradecer de manera especial a los directores y al personal involucrado en el reporte del SIP 2000 de los diferentes Establecimientos de Salud del país, que brindaron facilidades para el recojo de la información.

Contribuciones de autoría: Todos los autores participaron en la redacción del artículo, revisión crítica y aprobación de la versión final, GSA, JP, LH, JPA participaron en la recolección de resultados y análisis de datos.

Fuentes de financiamiento: Instituto Nacional de Salud.

Conflictos de interés: Ninguno declarado por los autores.

\section{REFERENCIAS BIBLIOGRÁFICAS}

1. Harris SR. Measuringhead circumference: Update on infant microcephaly. Can Fam Physician. 2015;61(8):680-4.

2. Dolk $H$. The predictive value of microcephaly during the first year of life for mental retardation at seven years. Dev Med Child Neurol. 1991;33(11):97483. doi: $10.1111 / \mathrm{j} .1469-8749.1991$. tb14813.x

3. World Health Organization. Declaración de la OMS sobre la primera reunión del Comité de Emergencia del Reglamento Sanitario Internacional (2005) sobre el virusdelZikayel aumento delos trastornos neurológicos y las malformaciones congénitas [Internet]. Geneva: World Health Organization; 2005. [citado 15 de septiembre de 2018]. Disponible en: http://www.who.int/es/news-room/ detail/01-02-2016-who-statement-onthe-first-meeting-of-the-internationalhealth-regulations-(2005)-(ihr-2005)emergency-committee-on-zika-virusand-observed-increase-in-neurologicaldisorders-and-neonatal-malformations

4. Steele A, Johnson J, Nance A, Satterfield R, Alverson CJ, Mai C. A quality assessment of reporting sources for microcephaly in Utah, 2003 to 2013. Birt Defects Res A Clin Mol Teratol. 2016;106(11):983-8. doi: 10.1002/bdra.23593.

5. Soares de Araújo JS, Regis CT, Gomes RGS, Tavares TR, Rocha dos Santos
C, Assunção PM, et al. Microcephaly in north-east Brazil: a retrospective study on neonates born between 2012 and 2015. Bull World Health Organ. 2016;94(11):835-40. doi: 10.2471/ BLT.16.170639.

6. Holden KR. Heads you win, tails you lose: measuring head circumference. Dev Med Child Neurol. 2014;56(8):705. doi: 10.1111/dmcn.12449.

7. Achee NL, Gould F, Perkins TA, Reiner RC, Morrison AC, Ritchie SA, et al. A critical assessment of vector control for dengue prevention. PLoS Negl Trop Dis. 2015;9(5):e0003655. doi: 10.1371/ journal.pntd.0003655.

8. von der Hagen M, Pivarcsi M, Liebe J, von Bernuth $\mathrm{H}$, Didonato N, Hennermann $\mathrm{JB}$, et al. Diagnostic approach to microcephaly in childhood: a two-center study and review of the literature. Dev Med Child Neurol. 2014;56(8):732-41. doi: $10.1111 / \mathrm{dmcn} .12425$.

9. Effio H, Cubas R, Pozadas G, Pizarro M. Microcefalia: estudio retrospectivo en el Hospital del Niño-Lima / Microcephaly: retrospective study in Hospital del NiñoLima. Diagnóstico Perú. 1987;20(1):18-24.

10. Orioli IM, Dolk H, Lopez-Camelo JS, Mattos D, Poletta FA, Dutra MG, et al. Prevalence and clinical profile of microcephaly in South America pre-
Zika, 2005-14: prevalence and casecontrol study. BMJ. 2017;359:j5018. doi: 10.1136/bmj.j5018.

11. Santos-Antonio G, Huamán-Espino L, Aparco JP, Pillaca J. Asociación entre el control prenatal y peso-longitud de los recien nacidos en establecimientos de salud nivel II y III del Ministerio de Salud periodo 2008-2012. Lima: Instituto Nacional de Salud; 2013.

12. Ministerio de Salud. Guía Técnica para la Categorización de Establecimientos del Sector Salud [Internet]. Lima: MINSA; 2014. Disponible en: ftp://ftp2.minsa. gob.pe/descargas/03esn/dir_ese/ RM076_2014_MINSA.pdf

13. Organización Panamericana de la Salud. Lineamientos preliminares de vigilancia de microcefalia en recién nacidos en entornos con riesgo de circulación de virus Zika. [Internet]. Washington DC: Organización Panamericana de la Salud; 2016 [citado 16 de septiembre de 2018]. Disponible en: https://www.paho.org/ hq/dmdocuments/2016/lineamientosprov-vigilancia-microcefalia.pdf

14. Fenton TR, Kim JH. A systematic review and meta-analysis to revise the Fenton growth chart for preterm infants. BMC Pediatr. 2013;13:59. doi: 10.1186/14712431-13-59.

15. Muñiz Landeros CE. Neurología clínica de Rangel Guerra. 1edicion. México, D.F: El Manual Moderno; 2015. 
16. Spong CY. Defining «term» pregnancy: recommendations from the Defining «Term» Pregnancy Workgroup. JAMA. 2013;309(23):2445-6. doi:10.1001/ jama.2013.6235.

17. Landis JR, Koch GG. The Measurement of Observer Agreement for Categorical Data. Biometrics. 1977;33(1):159-74.

18. Morris JK, Rankin J, Garne E, Loane M, Greenlees R, Addor M-C, et al. Prevalence of microcephaly in Europe: population based study. BMJ. 2016;354:i4721. doi: 10.1136/bmj.i4721.

19. Hansen M, Armstrong PK, Bower C, Baynam GS. Prevalence of microcephaly in an Australian population-based birth defects register, 1980-2015. Med J Aust. 2017;206(8):351-6. doi: 10.5694/ mja16.00966.

20. Cragan JD, Isenburg JL, Parker SE, Alverson CJ, Meyer RE, Stallings EB, et al. Population-Based Microcephaly Surveillance in the United States, 2009 to 2013: An Analysis of Potential Sources of Variation. Birt Defects Res A Clin Mol Teratol. 2016;106(11):972-82. doi: 10.1002/bdra.23587.

21. Bhide P, Kar A. Birth prevalence of microcephaly in India. Bull World Health Organ. 2016 Feb. Doi 10.2471/ BLT.16.172080.

22. Imaizumi Y. Prevalence and Mortality Rates of Microcephaly in Japan, 19691992. Congenit Anom. 1994;34(2):131-7. doi: 10.1111/j.1741-4520.1994.tb00796.x

23. Jaenisch $T$, Rosenberger KD, Brito $C$, Brady O, Brasil P, Marques ET. Risk of microcephaly after Zika virus infection in Brazil, 2015 to 2016. Bull World Health Organ. 2017;95(3):191-8. doi: 10.2471/ BLT.16.178608.

24. Candelo E, Caicedo G, Feinstein M, Pachajoa H. Microcephaly in Colombia before the Zika outbreak: A systematic literature review. Biomedica. 2018;38(0):127-134. doi: 10.7705/ biomedica.v38i0.4413.

25. Marinho F, Araújo VEM de, Porto DL, Ferreira HL, Coelho MRS, Lecca RCR, et al. Microcephaly in Brazil: prevalence and characterization of cases from the Information System on Live Births (Sinasc), 2000-2015. Epidemiol Serv Saude. 2016;25(4):701-12. doi: 10.5123/ S1679-49742016000400004.

26. Rick A-M, Domek G, Cunningham M, Olson D, Lamb M, Jimenez-Zambrano A, et al. Baseline microcephaly prevalence in rural Guatemala: implications for neonatal screening for congenital Zika virus infection. Lancet Glob Health. 2017;5:S16. doi: 10.1016/S2214109X(17)30123-7.

27. Quintó L, García-Basteiro AL, Bardají A, González R, Padilla N, MartinezEspinosa FE, et al. The Challenge of Assessing Microcephaly in the Context of the Zika Virus Epidemic. J Trop Pediatr. 2017. doi: 10.1093/tropej/fmx015.

28. González R, Mombo-Ngoma G, Ouédraogo S, Kakolwa MA, Abdulla $S$, Accrombessi $M$, et al. Intermittent preventivetreatment of malariain pregnancy with mefloquine in HIV-negative women: a multicentre randomized controlled trial. PLoS Med. 2014;11(9): e1001733. doi: 10.1371/journal.pmed.1001733.

29. Bardají A, Martínez-Espinosa FE, Arévalo-Herrera M, Padilla N, Kochar $S$, Ome-Kaius $\mathrm{M}$, et al. Burden and impact of Plasmodium vivax in pregnancy: A multi-centre prospective observational study. PLoS Negl Trop Dis. 2017;11(6):e0005606. doi: 10.1371/ journal.pntd.0005606.

30. Leviton A, Holmes LB, Allred EN, Vargas J. Methodologic issues in epidemiologic studies of congenital microcephaly. Early Hum Dev. 2002;69(1-2):91-105. doi: 10.1016/s0378-3782(02)00065-8.

31. Júlíusson PB, Roelants M,Hoppenbrouwers K, Hauspie R, Bjerknes R. Growth of Belgian and Norwegian children compared to the WHO growth standards: prevalence below -2 and above $+2 \mathrm{SD}$ and the effect of breastfeeding. Arch Dis Child. 2011;96(10):916-21. doi: 10.1136/ adc.2009.166157.

Correspondencia: Gabriela Ruth Santos Antonio. Dirección: Jr. Tizón y Bueno 276 Jesús María. Lima, Perú. Teléfono: (+51) 748-0000 anexo 6626. Correoelectronico:gsantos@ins.gob.pe 